\title{
Performance Evaluation of Malaria HRP-2 Rapid Diagnostic Test among Febrile Patients with Malaria in Iwo, Osun State, Nigeria
}

\author{
Oladosu Oladipo $\mathrm{O}^{1,2^{*}}$, Adedokun Victoria $\mathrm{A}^{1}$, Adeniyi Akinkunle $\mathrm{V}^{1}$ and Oyibo Wellington $\mathrm{A}^{2}$
}

${ }^{1}$ Pure and Applied Biology Programme, Bowen University, Iwo, Osun State, Nigeria

${ }^{2}$ ANDI Centre of Excellence for Malaria Diagnosis, College of Medicine, University of Lagos, Nigeria

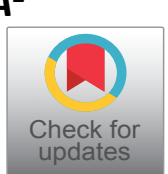

*Corresponding author: Oladosu Oladipo O, Pure and Applied Biology Programme, Bowen University, Iwo-Osun, Osun State, Nigeria, Tel: 234-8034049078

\begin{abstract}
Diagnosis of malaria is a fundamental component of malaria case management. Parasitological confirmation of malaria before treatment was recommended for endemic countries. Microscopy diagnosis of malaria, the gold standard as constraints such as: level of expertise, quality of equipment and reagents may lead to misdiagnosis. The histidine rich protein 2 (HRP-2) based rapid diagnostic test (RDT) detects Plasmodium falciparum only. We therefore evaluated the performance of malaria HRP-2 rapid tests among febrile patients suspected to have malaria in Iwo, Osun state Nigeria. A cross-sectional study was conducted on patients with fever or symptoms suggestive of malaria at the Outpatient's Department of a University Medical Centre in Iwo, Osun State, Nigeria. Malaria diagnosis was performed with the malaria HRP-2-based RDT and microscopy for every participant. Stained Thick and thin films prepared on the same slide, stained with $3 \%$ Giemsa stain and evaluated for detection, speciation and parasite density per patient.. Malaria RDT results were read as positive or negative. Of the 289 febrile patients assessed between October 2016 to August 2017, the number positive by Malaria RDT and microscopy were $102(35.3 \%)$ and 98 (33.9\%) respectively. Parasite density ranged from $76 \mathrm{p} / \mu \mathrm{L}$ to $165,333 \mathrm{p} / \mu \mathrm{L}$ of blood. Overall, the sensitivity, specificity, positive and negative predictive values were: $96.08 \%, 97.95 \%, 96.08 \%$ and $97.95 \%$ respectively. This study showed malaria RDT could be a reliable option to microscopy for effective case management of uncomplicated falciparum malaria in febrile patients in Iwo, Nigeria.
\end{abstract}

\section{Keywords}

Malaria, HRP-2, Plasmodium falciparum, mRDT, Sensitivity and Specificity

\section{Introduction}

An estimated 229 million cases of malaria burden occurred worldwide in 87 malaria endemic countries with WHO African region having an estimated 215 million malaria cases, accounted for about $94 \%$ of cases in 2019 [1]. Twenty-nine countries accounted for $95 \%$ of malaria cases globally, with Nigeria having the highest, $27 \%$ [1]. A study by Nigeria Demographic and Health Survey (NDHS) recorded a prevalence of $23 \%$ [2]. Prompt diagnosis of malaria disease remains the basis for improved case management. Presumptive treatment of malaria based on clinical diagnosis and non-adherence to malaria parasitological confirmed negative results is still a challenge in many health facilities in Nigeria. Over diagnosis and misdiagnosis of malaria, still practiced around the world, results in unnecessary use of antimalarial drugs, exaggeration of the burden of the disease, delay of appropriate treatment and sometimes death due to non-malaria causes of fever [3].

Laboratory diagnosis of malaria is an essential component of malaria case management. It involves control and elimination strategy that promises accurate diagnosis and treatment with effective medication. It is difficult to determine if the cause of febrile illness is due to malaria based on clinical presentation alone. Parasitological confirmation of suspected malaria cases before initiating anti-malarial treatment was recommended for malaria endemic countries [4]. Diagnostic accuracy should significantly improve the quality of care and ensure a rational and appropriate use of antimalarial drugs.

Citation: Oladosu OO, Adedokun VA, Adeniyi AV, Oyibo WA (2021) Performance Evaluation of Malaria HRP-2 Rapid Diagnostic Test among Febrile Patients with Malaria in Iwo, Osun State, Nigeria. Int J Trop Dis 4:046. doi.org/10.23937/2643-461X/1710046 Accepted: March 29, 2021; Published: March 31, 2021

Copyright: (C) 2021 Oladosu OO, et al. This is an open-access article distributed under the terms of the Creative Commons Attribution License, which permits unrestricted use, distribution, and reproduction in any medium, provided the original author and source are credited. 
Microscopy diagnosis of malaria remains the gold standard for malaria diagnosis because it is inexpensive, has high sensitivity and allows Plasmodium species identification and quantification of parasite density $[5,6]$. However, light microscopy being the gold standard method is time-consuming and requires highly trained personnel. Microscopy is reported to detect about $75 \%$ of malaria infections in high transmission areas, whereas in low transmission areas this method has been reported to miss up to $88 \%$ of infections [7]. Furthermore, the level of expertise of technicians, quality of the equipment, and workload may lead to misdiagnosis, inaccurate estimates of parasite density and species differentiation [8]. The implementation of rapid diagnostic test has contributed to the timely diagnosis and management of malaria in some endemic countries and reduction in overtreatment of malaria disease. The current use of rapid diagnostic tests (RDTs) to detect antigens constitutes an important forward step in the diagnostic strategy because it allows parasitological diagnosis even in areas where it is impossible to have good microscopy services [9]. RDTs detect malaria antigens, not malaria parasites, this give RDT an added advantage in the ability to diagnose malaria in patients with lowgrade parasitaemia.

It has been reported that with the availability of RDTs, clinicians treat febrile presentations with anti-malarial drugs, even when the result of the RDT is negative for malaria parasite antigen. A study showed that about half of all negative RDT patients were prescribed anti-malarial drugs [10-12]. Malaria RDTs are based on the detection of one of the following three antigens, histidine-rich protein-2 (HRP2), lactate dehydrogenase (LDH) and aldolase that show the differences in the sensitivity and specificity seen in RDT test kits [13]. The majority of commercially available malaria RDTs, target HRP II $[13,14]$. HRP-II is more sensitive antigen for detecting Plasmodium falciparum infections than any other antigens [15]. The HRP-II test kit is the most preferred for detecting the presence of $P$. falciparum in the blood and it is the most commonly used in Nigeria because HRP-II is only peculiar to $P$. falciparum, which is the most common Plasmodium species in Nigeria. Other non- $P$. falciparum species have $<2 \%$ prevalence.

The performance of HRP2- immunochromatography-based RDTs that is specific to Plasmodium falciparum [16] is affected by the detection of persistent antigenicity that may remain in circulation for about 28 days after parasite clearance, which leads to false positivity and may overestimate prevalence $[5,17,18]$. Deletions or mutations within the Pfhrp-2 gene and the prozone effect may lead to false negatives [19-22].

Proper diagnosis of malaria parasites in all suspected cases before treatment with artemisinin combination therapy (ACTs) is important to reduce over prescription and reduce onset of resistance. The malaria microscopy diagnosis, the primary tool for scaling up malaria diagnosis as its limitations, that include: The level of expertise of technicians, quality of the equipment, and workload that may lead to misdiagnosis [8]. The use of malaria RDTs though, not a replacement for malaria microscopy, possess qualities that address many of the limitations of microscopy. This makes the use of RDT a more feasible strategy for expanding access to diagnosis [17,23-25]. Malaria microscopy, which is the gold standard for detection of Plasmodium parasites have been on the decrease due to challenges such as the level of expertise, quality of the reagents, equipment, the tedious process involve and since the introduction of malaria RDTs in many health care centers including secondary facilities in Nigeria. This study is therefore imperative to evaluate the reliability of malaria RDTs in diagnosis of febrile individuals suspected to have malaria in Iwo, Osun state Nigeria.

\section{Materials and Methods}

\section{Study population and designs}

This was a cross-sectional study, conducted on 289 individuals, who presented with fever or with signs and symptoms suggestive of malaria at the Outpatient's Department (OPD) of Bowen University Hospital Iwo, Osun State. Bowen University is located in the urban setting of the small city of Iwo (population range of 250,000 499,999 inhabitants), Osun State. Bowen University is located in N 40 $0^{\prime} 19.152^{\prime \prime}$, W $83^{\circ} 0^{\prime} 34.0308^{\prime \prime}$ (Figure 1). The individuals were referred to laboratory for malaria testing based on the clinicians' prescription. The study was conducted from October 2016 to August 2017. The health care facility attends to about 5,000 students and some of the inhabitants making use of the facility. The health care facility is not only been utilised by the students, outpatient services are also rendered to the inhabitants of Iwo community.

\section{Study samples and inclusion criteria}

The recruited individuals include students of Bowen University Iwo and some inhabitants of Iwo community of different age, sex and educational status attending the facility. Venous blood samples were collected into an EDTA bottles for laboratory analysis majorly for MP and Widal test. Malaria infection is defined as individual with evidence of malaria parasite in blood either by testing using microscopy or malaria RDT. Participants were enrolled in the study if they met the following inclusion criteria: documented fever at presentation as suggested by the physicians, absence of danger signs of complicated/ severe malaria/known serious chronic disease and willingness to consent to participate. Those that presented with signs of complicated malaria namely: convulsions/ coma, prostration, severe vomiting, etc were excluded and these were managed appropriately or referred. Pregnant women were also excluded from the study.

Questionnaire that captured age, sex, medications taken before presentation, history of fever in the last 24 


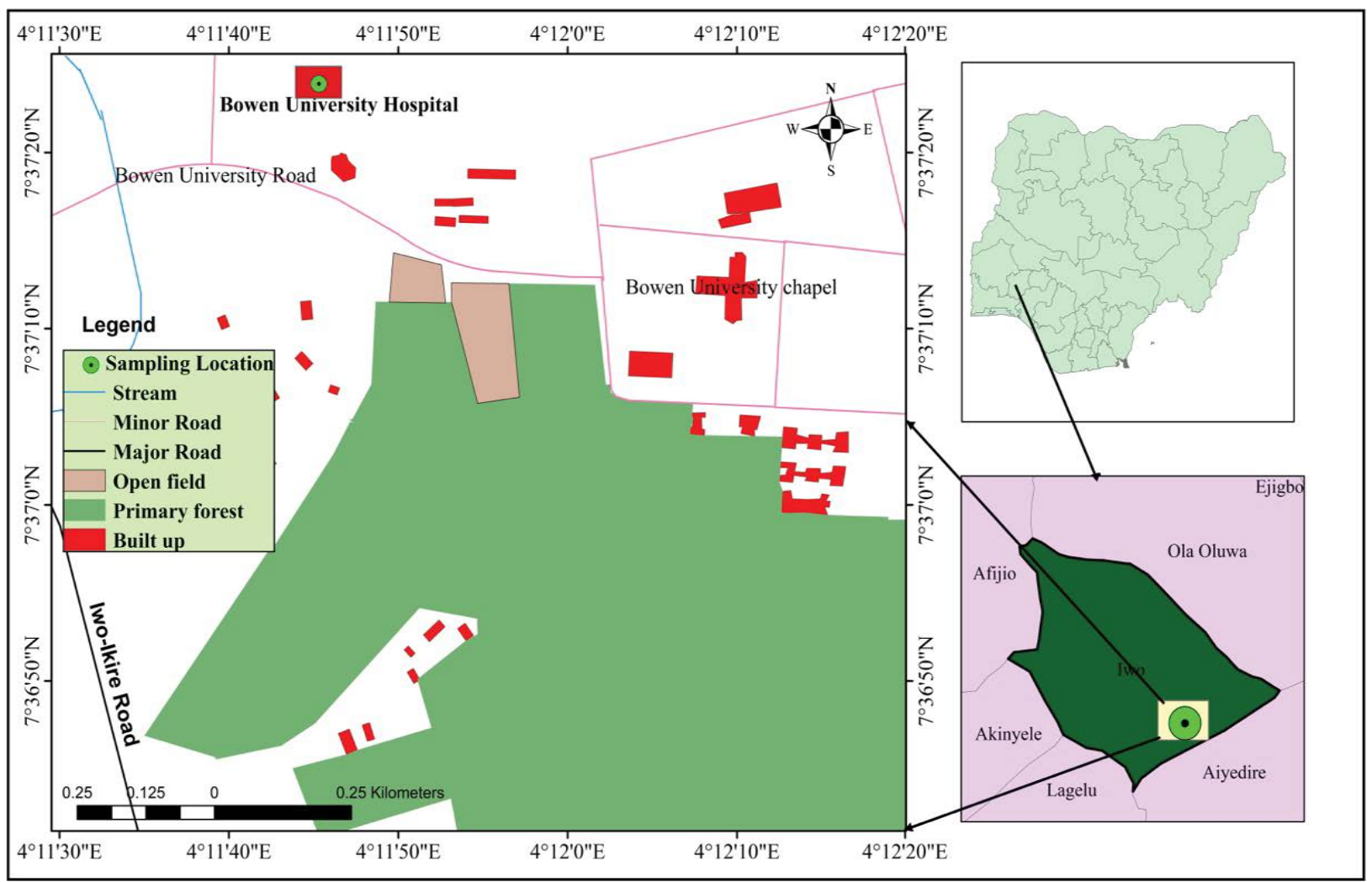

Figure 1: Map of the study area showing the sampling location (Inset: maps of Osun State, Nigeria and showing the location of the study area).

hours, other presenting symptoms, knowledge, attitude and practice was completed before blood test was carried out for malaria RDT and microscopy. Every individual with positive $\mathrm{mRDT}$ received standard care provided in the health facilities whether they participated in the study or not.

\section{Sample collection}

About $2 \mathrm{~mL}$ of blood samples were collected from each individual into and EDTA bottle for malaria diagnosis test and for other lab tests, as requested by the physicians. About $20 \mu \mathrm{L}$ of the EDTA blood were used for mRDT and smear making. Briefly, $5 \mu \mathrm{L}$ of blood were used for mRDT testing and about $2 \mu \mathrm{L}$ and $12 \mu \mathrm{L}$ were used for both thin and thick films respectively. Thick and thin blood films were prepared on the same slide. The blood films were then allowed to air dry before staining.

\section{Rapid diagnosis}

The blood samples collected by finger prick were assayed using SD-Bioline Malaria HRP-II Antigen \{LOT NO: 1811-2\}. It is an immunochromatographic test that detects the presence of Plasmodium falciparum. The test uses approximately $5 \mu \mathrm{L}$ of blood and is readable after 15 minutes following the manufacturer's instructions (Standard Diagnostics, Republic of Korea). The results were recorded in comparison with the control line as positive if a unique Pf line appears indicating $P$. falciparum infection. All tests were performed and results were interpreted and recorded following the manufacturer's instructions.

\section{Staining of Malaria Blood Films with Giemsa}

The thin films were fixed in methanol for about a second by dipping the thin smear part only into methanol. It is then allowed to dry and placed on the staining rack. The thick and thin smears were then stained with 3\% Giemsa working solution for 45 minutes [26]. The stain was then washed off with the distilled water and the slides are allowed to air dry at room temperature, after which they are then stored in the slide box prior to microscopic examination.

\section{Microscopic examination of stained thick and thin blood smears}

The parasite density was computed as the number of parasites per 500 leucocytes on a thick film and reported as parasites per microlitre of blood assuming an average white blood-cell count of $8000 / \mu \mathrm{L}$. The mRDT test and microscopy reading were done simultaneously for each individual. The mRDT results were however blinded to the microscopists. The two microscopists that read the slides were blinded to each other results. Stained slides were examined under the light microscope using $\times 100$ objective lens (immersion oil). A slide was considered negative after 100 high power fields (HPF) have been examined without a malaria parasite. Another microscopist, the second reader, was made to reread each slide. A third reader, who served as the tiebreaker, reread parasitaemia $>20 \%$ discordance between the two readers. However, parasite counts of $<20 \%$ discordance between the first and second readers were accepted and 
the mean parasite count taken to compute the parasite density or parasitaemia for every positive individual. Certified malaria microscopists read slides.

\section{Ethical approval and informed consent}

Bowen University Teaching Hospital Research Ethics Committee Ogbomosho, Oyo State and the Osun State Hospitals' Management Board approved the protocol for this study. Consent forms were given to all participants and were duly filled and signed. All procedures were conducted with good clinical and laboratory practices. All patients with confirmed malaria infection (Uncomplicated and asymptomatic infections) based on the RDT and microscopy results were treated with artemisinin combination therapy (ACTs).

\section{Sample size}

The sample size of 272 was calculated using pilot study that was previously carried out with a prevalence of $23 \%$ in Iwo. The sample size was determined using a statistical formula $\left[n=Z^{2} p(1-p) / d^{2}\right]$ considering $95 \%$ confidence interval $(z=1.96)$ and a level of precision (d) of $5 \%$.

\section{Data analysis}

Data was entered on Excel 2013 and analysed using SPSS 20.0 version statistical software. The questionnaire was administered by a trained field scientist and it was validated by three experts taking into consideration the content of the instrument, clarity, appropriateness of the language and suitability. Some of the content was reworded and it covers the major areas of the study. The number of individuals positive to malaria is the dependent variable, while test method (mRDT or microscopy) is independent variables for this study. Data were analysed using frequency and percentages and results presented in tables. Sensitivity was defined as the pro- portion of true malaria cases (positive blood smears) that were correctly identified by positive RDTs whereas specificity was the proportion of true negative malaria cases (negative blood smears) that were correctly identified by negative RDTs. Positive predictive value was the proportion of true malaria cases (positive blood smears) among the individuals with the positive RDTs. Negative predictive value was the proportion of true negative malaria cases (negative blood smears) among the total number of negative RDT tests. Chi-square was used to determine whether there is an agreement between the results of the two tests. $P<0.05$ was considered statistically significant. Taking blood slide microscopy as the gold standard, the performance of the Rapid Diagnostic Test was compared to it by computing the sensitivity, specificity, the negative predictive value, and the positive predictive value of the test [27].

\section{Results}

A total of 289 febrile individuals were sampled for malaria parasite in Bowen University Hospital, Iwo Osun State, Nigeria from October 2016 to August 2017. The study population comprised of $184(63.7 \%)$ females and 105 (36.3\%) males. The ages of the participants ranged from 15 to 50 years and the mean age was 21 years. The total number of individuals positive for malaria RDT was $102(35.3 \%)$ and $98(33.9 \%)$ individuals were positive for microscopy diagnosis with no significance different $(p>$ 0.793) (Table 1).

The participants had parasite density ranging from 76 to 165,333 parasites/ $\mu \mathrm{L}$. The parasite density was grouped to show the different level of parasitaemia in different age groups (Table 2). Majority of the species diagnosed is Plasmodium falciparum (98.98\%) with only one Plasmodium ovale (1.02\%). Higher parasitaemia level was noticed majorly in the younger age groups

Table 1: Frequency of age groups and malaria microscopy and RDT results.

\begin{tabular}{|l|l|l|l|l|l|}
\hline $\begin{array}{l}\text { Age Groups } \\
\text { (years) }\end{array}$ & Frequency & \multicolumn{3}{l|}{ RDT (\%) } & \multicolumn{3}{l|}{ Microscopy (\%) } \\
\cline { 2 - 6 } & Positive & Negative & Positive & Negative \\
\hline $15-25$ & $256(88.6)$ & $89(34.8)$ & $167(65.2)$ & $85(33.2)$ & $171(66.8)$ \\
\hline $26-36$ & $17(5.9)$ & $6(35.3)$ & $11(64.7)$ & $9(52.9)$ & $8(47.1)$ \\
\hline $36-45$ & $9(3.1)$ & $4(44.4)$ & $5(55.6)$ & $2(22.2)$ & $7(77.8)$ \\
\hline $46-50$ & $7(2.4)$ & $3(42.9)$ & $4(57.1)$ & $2(28.6)$ & $5(71.4)$ \\
\hline Total & 289 & $102(35.3)$ & $187(64.7)$ & $98(33.9)$ & $191(66.1)$ \\
\hline
\end{tabular}

Table 2: Parasitaemia groups in different age groups.

\begin{tabular}{|l|l|l|l|l|l|l|}
\hline \multirow{2}{*}{$\begin{array}{l}\text { Age Groups } \\
\text { (years) }\end{array}$} & \multicolumn{4}{|c|}{ Frequency of Parasitaemia Groups (\%) } & Total (\%) \\
\cline { 2 - 7 } & $1-1000 \mathrm{p} / \mu \mathrm{L}$ & $1001-10000 \mathrm{p} / \mu \mathrm{L}$ & $10001-40000 \mathrm{p} / \mu \mathrm{L}$ & $40001-100000 \mathrm{p} / \mu \mathrm{L}$ & $100001-200000 \mathrm{p} / \mu \mathrm{L}$ & \\
\hline $15-25$ & $14(87.5)$ & $28(84.8)$ & $23(82.1)$ & $15(93.8)$ & $5(100)$ & $85(86.7)$ \\
\hline $26-36$ & $2(12.5)$ & $4(12.1)$ & $3(10.7)$ & $0(0.0)$ & $0(0.0)$ & $9(9.2)$ \\
\hline $36-45$ & $0(0.0)$ & $0(0.0)$ & $1(3.6)$ & $1(3.6)$ & $0(0.0)$ & $2(2.0)$ \\
\hline $46-55$ & $0(0.0)$ & $1(3.6)$ & $1(3.6)$ & $0(0.0)$ & $0(0.0)$ & $2(2.0)$ \\
\hline Total & $16(16.3)$ & $33(39.8)$ & $28(21.4)$ & $16(16.3)$ & $5(6.2)$ & 98 \\
\hline
\end{tabular}


(15-25 years).

\section{Performance of RDT using microscopy as a refer- ence test}

The sensitivity and specificity of the malaria RDT was $96.08 \%$ and $97.95 \%$ respectively, with corresponding positive and negative predictive values (PPV and NPV) of $96.08 \%$ and $97.95 \%$ (Table 3 ). Malaria RDT sensitivity and specificity seemed to be higher in the lowest age group in this study (15-25 years).

Different presenting symptoms were observed in the different age groups, with those in the youngest age groups presenting majorly with fever (93.2\%), while few of them (16\%) presented with convulsion (Table 4). Convulsion was only reported in age 15-25 years.

One hundred and seventy-five (175) participants had records for use of ITNs. A total of 40 (22.8\%) participants made use of the ITNs at home/hostel with 14 (35.0\%) having malaria infection and $26(65.0 \%)$ were malaria negative. Many of the participants did not make use of ITNs with 34 (25.2\%) having malaria infection and $135(77.1 \%)$ were malaria negative $(p=0.001)$.

The record of participants previous antimalarial treatment showed that majority of them used monotherapy for malaria treatment with majority of them taking Artesunate $(29.4 \%)$, followed by Sulphadoxine-pyrimethamine (SP) (17.6\%). Only few of the participants (28.0\%) took the recommended antimalarial drugs, Arteminisin Combination Therapy, during the previous malaria treatment (Table 5). More females were seen to have taken monotherapy during their previous malaria treatment.

\section{Discussion}

This study evaluated the performance of malaria rapid diagnostic test using malaria microscopy as gold standard in the diagnosis of febrile individuals suspect- ed to have malaria attending Bowen University Hospital, Iwo, Osun state Nigeria. The high sensitivity and specificity results reported in this study showed the reliability of malaria RDT in malaria diagnosis, especially in rural arears and where there is no expert malaria microscopist to carry out proper malaria diagnosis. Malaria diagnosis result showed that $33.9 \%$ of individuals tested where positive by malaria microscopy. A previous study done in Iwo, Osun State Nigeria reported that out of 733 patients recruited, 684 (93.3\%) had P. falciparum only, $22(3.0 \%)$ had $P$. malariae only and $27(3.7 \%)$ had both $P$. falciparum and $P$. malariae [28].

The high sensitivity, specificity, PPV and NPV reported for malaria RDT in this study could help to improve malaria diagnosis, since the RDT is unlikely to miss out the infected and non-infected individuals. The high PPV in this study RDT suggest that any individual with positive RDT test would probable have malaria with respect to malaria microscopy. Although, predictive values rely more on disease prevalence in a given population, they give practical usefulness on test information. According to the result in this study, any individual who will be tested with a positive result using this RDT is highly likely to be malaria positive. It therefore means that every positive result should be considered with no doubt be treated immediately. In essence, it will reduce over treatment that leads to emergence of antimalarial resistance. Identical study revealed that sensitivity, specificity, PPV and NPV of mRDT at $95 \% \mathrm{Cl}$ was $97.6 \%, 97.4 \%$, $91.0 \%$ and $99.3 \%$ respectively [29]. A Similar study, where SD Bioline Pf antigen HRP II was used, have sensitivity, specificity, PPV, and NPV of $100 \%, 98 \%, 88 \%$, and $100 \%$, respectively, among symptomatic children aged $<5$ years in a hospital in a mesoendemic area in Markafi in Kaduna state, Nigeria [30]. This result also compares favourably with that of other studies [12,3133] that reported a variable sensitivity of $84.2 \%-100 \%$ and specificity of $82.8 \%-98.5 \%$. The sensitivity of this test was also close to that of Iran (100\%), which made

Table 3: Performance characteristic of malaria RDT in different age groups.

\begin{tabular}{|c|c|c|c|c|c|c|}
\hline $\begin{array}{l}\text { Age Groups } \\
\text { (years) }\end{array}$ & $\begin{array}{l}\text { RDT } \\
\text { Positivity (\%) }\end{array}$ & $\begin{array}{l}\text { Microscopy } \\
\text { Positivity (\%) }\end{array}$ & $\begin{array}{l}\text { Sensitivity } \\
{[95 \% \mathrm{Cl}](\%)}\end{array}$ & $\begin{array}{l}\text { Specificity } \\
{[95 \% \mathrm{Cl}](\%)}\end{array}$ & $\begin{array}{l}\text { Positive } \\
\text { Predictive Value } \\
\text { (PPV) } \\
{[95 \% \mathrm{Cl}](\%)}\end{array}$ & $\begin{array}{l}\begin{array}{l}\text { Negative } \\
\text { Predictive Value } \\
\text { (NPV) }\end{array} \\
{[95 \% \mathrm{Cl}](\%)}\end{array}$ \\
\hline $15-25$ & 89 (87.3) & $85(86.7)$ & $\begin{array}{l}95.51 \\
{[88.89-98.76]}\end{array}$ & $\begin{array}{l}97.71 \\
{[94.25-99.37]}\end{array}$ & $\begin{array}{l}95.43 \\
{[88.78-98.21]}\end{array}$ & $\begin{array}{l}97.76 \\
{[94.35-99.13]}\end{array}$ \\
\hline $26-36$ & $6(5.9)$ & $9(9.2)$ & $\begin{array}{l}75.00 \\
{[42.81-94.51]}\end{array}$ & $\begin{array}{l}72.73 \\
{[39.03-93.98]}\end{array}$ & $\begin{array}{l}75.00 \\
{[51.99-89.26]}\end{array}$ & $\begin{array}{l}72.73 \\
{[48.40-88.34]}\end{array}$ \\
\hline $36-45$ & $4(3.9)$ & $2(2.0)$ & $\begin{array}{l}50.00 \\
{[6.76-93.24]}\end{array}$ & $\begin{array}{l}77.78 \\
{[39.99-97.19]}\end{array}$ & $\begin{array}{l}50.00 \\
{[17.27-82.73]}\end{array}$ & $\begin{array}{l}77.78 \\
{[55.29-90.83]}\end{array}$ \\
\hline $46-50$ & $3(2.9)$ & $2(2.0)$ & $\begin{array}{l}66.67 \\
{[9.43-99.16]}\end{array}$ & $\begin{array}{l}83.33 \\
{[35.88-99.58]}\end{array}$ & $\begin{array}{l}66.67 \\
{[21.98-93.42]}\end{array}$ & $\begin{array}{l}77.78 \\
{[39.99-97.19]}\end{array}$ \\
\hline Total & $102(35.3)$ & $98(33.9)$ & $\begin{array}{l}96.08 \\
{[90.26-98.92]}\end{array}$ & $\begin{array}{l}97.95 \\
{[94.83-99.44]}\end{array}$ & $\begin{array}{l}96.08 \\
{[90.27-98.48]}\end{array}$ & $\begin{array}{l}97.95 \\
{[94.81-99.21]}\end{array}$ \\
\hline
\end{tabular}




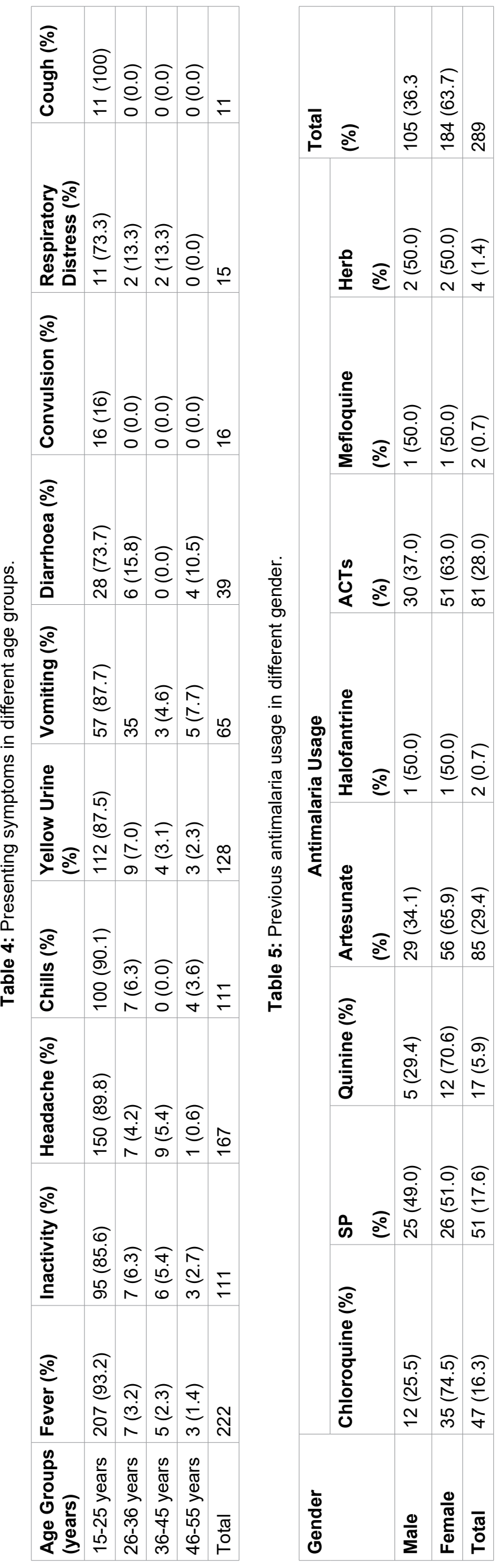

use of Biotec Mal Pv/Pf rapid device from a similar manufacturer [34]. In contrast to this study, sensitivity and specificity of the RDT was found to be $78.4 \%$ and $97.6 \%$ respectively while the PPV and the (NPV) were found to be $97.3 \%$ and $80.1 \%$ respectively [35]. The sensitivity obtained at present (96.08\%) was similar to a study in Ibadan South-western Nigeria (93.1\%) [36], but was much higher than what was reported in Lagos (69.9\%) [37]. Variations in test sensitivity between all these studies may be due to differences factors, such as: Low levels of parasitaemia [38], inherent limitation of the device, mutation or deletion of the gene encoding the HRP-II, and storage conditions [38,39] or lack of adequate quality control and assurance systems for malaria microscopy that may result in over diagnosis [40].

False positive result in malaria RDT could be due to a lot of factors. The rheumatoid factor cross-reacting in the blood could generate a false positive test line but the replacement of IgG and IgM helps in reducing this problem [41,42]. False positive test may be due to cross-reactivity with heterophile antibodies [43]. Another cause of false positive results could be due to the persistence of HRP-II antigen in the blood, delayed clearance of circulating antigens and also the presence of gametocytes that produce the antigens may be reasons for persistence [44]. HRP-II persistence may occur up to 28 days even when there is rapid clearance of microscopically detectable parasite $[5,44,45]$. False negative test may result from mutation of the HRP-II gene [46].

The use of malaria rapid test is suggested to have largest impact with microscopy been widely accepted as the gold standard for malaria diagnosis. Its implementation has been reported to be cost effective [47]. Malaria RDT is a device that detects malaria antigen in a very small amount of blood usually 5-15 $\mu \mathrm{L}$ using immunochromatographic assay with monoclonal antibodies directed against the target parasite antigen and is been impregnated on a test strip [38]. The non-electricity requirement, easy operation and result guide interpretation are the advantages of it over microscopy. The threshold of detection of these rapid diagnostic tests is in the range of 100 parasites/ $\mu \mathrm{L}$ of blood (i.e. about $0.002 \%-0.1 \%$ parasitaemia) [48]. It is important to accurately diagnose malaria especially in malaria-endemic regions like Nigeria, because it will help to reduce overdiagnosis and overtreatment of malaria infections and limit antimalaria usage to only malaria parasite-based test true positives. Malaria RDT is mainly deployed to rural areas because of lack of manpower to in rural arears to carry out malaria microscopy. RDTs should be used to complement microscopy or alone, especially in secondary facilities where expert microscopy is unavailable or where there are a paucity of reagents for malaria microscopy.

The National Guideline for Diagnosis and Treatment of Malaria recommends the use of microscopy only in 
tertiary and secondary health facilities where there are functional laboratory services with qualified laboratory scientists [49]. Microscopy is the gold standard for malaria diagnosis, giving species and accurate amounts of parasite in the blood hence they are quantitative and at the same time qualitative [50]. Microscopy could be more sensitive than RDTs if done well by expert malaria microscopist with standard external quality assessment (EQA) programme for malaria microscopy feedback system. However, some of the constraints associated with it is that it requires expertise training and cannot perform in conditions such as absence of light and also the time needed to prepare the stained blood smear and to examine it microscopically is time consuming especially in cases of emergency [51,52]. A 10-day malaria microscopy training in Nigeria showed that many of the malaria microscopy diagnosis by the participants before the training were misdiagnosis as seen in their pre-test $(38.3 \%)$ and post test $(70.7 \%)$ results [53].

Many studies across Africa have reported misdiagnosis of malaria with microscopy that leads to false positive results $[54,55]$. A number of factors have been suggested to contribute to poor performance of routine malaria microscopy in Angola; these factors include the large number of thick blood smears to read in daily routine, lack of skilled microscopists, supervision, and maintenance of infrastructure [56]. To continue to depend on malaria microscopy for correct case management of malaria in Nigeria, where decline in malaria is being reported since the introduction of malaria intervention. Continuous malaria microscopy training including proficiency assessment of every laboratory staff involved in malaria microscopy diagnosis is pertinent. Laboratory supervision as well implementation of internal and/or external quality control is important to achieve improvement of malaria diagnosis by microscopy.

The most occurring symptom was fever, which occurred in 222 (76.8\%) participants but only 44 (44.9\%) among them were confirmed positive for malaria. However, most of the people who presented with fever were negative for malaria. Hence, proving that fever alone is not enough to clinical diagnose malaria infection. Fever symptom may be as a result of some other types of infection in the body system which may be viral, bacterial and parasitic infection such as common cold, urinary tract infections, meningitis, malaria, appendicitis among others [57].

Insecticides treated net (ITN) could be a powerful tool, which protect from mosquitoes' bite. The knowledge, attitude and practice of some of the individuals that participated in this study concerning the use of ITN showed that ITNs may be a protected tool against mosquitoes' bite. A high number of those that were malaria negative in this study reported to have been using ITNs regularly in contrast to those that were malaria positive. Insecticide-treated nets (ITNs) represent a powerful means of controlling malaria in Africa [58]. This shows that the use of good insecticide treated net will help to further reduce the incidence of malaria in these individuals.

\section{Conclusion}

This study showed the effectiveness of malaria RDT as an alternative method to microscopy for detection and management of $P$. falciparum in individuals with febrile illness in Nigeria. Although this study does not suggest that malaria RDT should replace microscopy as a diagnostic tool as malaria RDT cannot differentiate the species of Plasmodium and cannot quantify malaria parasitaemia. However, malaria RDT can be used to improve the quality of case management of malaria and appropriate treatment of confirmed malaria cases while avoiding indiscriminate administration of anti-malarial drugs for malaria-negative patients.

\section{Acknowledgement}

We acknowledge the support of the management and staff of Bowen University Hospital Iwo, Osun State in providing the platform for assessing the patients in this study. In addition, we thank the laboratory staff of Department of Biological Sciences for permission to use their laboratory for this study.

\section{Competing Interest}

The authors declare that they have no competing interest.

\section{References}

1. WHO (2020) World malaria report 2020: 20 years of global progress and challenges. World Health Organization, Geneva.

2. National Population Commission (NPC) [Nigeria] and ICF (2019). Nigeria Demographic and Health Survey 2018. Abuja, Nigeria, and Rockville, Maryland, USA: NPC and ICF.

3. Oladosu OO, Oyibo WA (2013) Overdiagnosis and Overtreatment of Malaria in Children that Presented with Fever in Lagos, Nigeria. Int. Sch. Res. Notices. 2013: ID 914675.

4. WHO (2015) Guidelines for the treatment of malaria. World Health Organization 2015 (3rd edition). WHO. Geneva.

5. Kyabayinze DJ, Tibenderana JK, Odong GW, Rwakimari JB, Counihan H (2008) Operational accuracy and comparative persistent antigenicity of HRP2 rapid diagnostic tests for Plasmodium falciparum malaria in a hyperendemic region of Uganda. Malar J 7: 221.

6. Chanie M, Erko B, Animut A, Legesse M (2011) Performance of CareStart ${ }^{\mathrm{TM}}$ Malaria Pf/Pv Combo test for the diagnosis of Plasmodium falciparum and Plasmodium vivax infections in the Afar Region, North East Ethiopia. Ethiop J Health Dev 25: 206-211.

7. Baiden F, Bruce J, Webster J, Tivura M, Delmini R, et al. (2016) Effect of test-based versus presumptive treatment of malaria in under-five children in rural Ghana--A cluster-randomised trial. PLoS One 11: e0152960.

8. Kilian AH, Metzger WG, Mutschelknauss EJ, Kabagambe 
G, Langi P, et al. (2000) Reliability of malaria microscopy in epidemiological studies: Results of quality control. Trop Med Int Health 5: 3-8.

9. WHO (2014) Recommended selection criteria for procurement of malaria rapid diagnostic tests. WHO Press, Geneva, Switzerland.

10. Reyburn $\mathrm{H}$, Mbakilwa $\mathrm{H}$, Mwangi R, Mwerinde $\mathrm{O}$, Olomi R, et al. (2007) Rapid diagnostic tests compared with malaria microscopy for guiding outpatient treatment of febrile illness in Tanzania: Randomised trial. BMJ 334: 403.

11. Skarbinski J, Ouma PO, Causer LM, Kariuki SK, Barnwell JW, et al. (2009) Effect of malaria rapid diagnostic tests on the management of uncomplicated malaria with artemether-lumefantrine in Kenya: A cluster randomized trial. Am J Trop Med Hyg 80: 919-926.

12. Ansah EK, Narh BS, Epokor M, Akanpigbiam S, Amu Quartey $A$, et al. (2010) Rapid testing for malaria in settings where microscopy is available and peripheral clinics where only presumptive treatment is available: A randomized controlled trial in Ghana. BMJ 340: 930.

13. Rosenthal PJ (2012) How do we best diagnose malaria in Africa? Am J Trop Med Hyg 86: 192-193.

14. Gatton ML, Dunn J, Chaudhry A, Ciketic S, Cunningham J, et al. (2017) Implications of parasites lacking Plasmodium falciparum histidine-rich protein 2 on malaria morbidity and control when rapid diagnostic tests are used for diagnosis. J Infect Dis 215: 1156-1166.

15. Gatton ML, Rees-Channer RR, Glenn J, Barnwell JW, Cheng Q, et al. (2015) Pan-Plasmodium band sensitivity for Plasmodium falciparum detection in combination malaria rapid diagnostic tests and implications for clinical management. Malar J 14: 115.

16. Bell D, Wongsrichanalai C, Barnwell JW (2006) Ensuring quality and access for malaria diagnosis: How can it be achieved? Nat Rev Microbiol 4: S7-S20.

17. Endeshaw T, Gebre T, Ngondi J, Graves PM, Shargie EB, et al. (2008) Evaluation of light microscopy and rapid diagnostic test for the detection of malaria under operational field conditions: A household survey in Ethiopia. Malar $J$ 7: 118 .

18. Ishengoma DS, Francis F, Mmbando BP, Lusingu JPA, Pamela Magistrado, et al. (2011) Accuracy of malaria rapid diagnostic tests in community studies and their impact on treatment of malaria in an area with declining malaria burden in north-eastern Tanzania. Malar J 10: 176.

19. Gillet $P$, Scheirlinck $A$, Stokx J, Weggheleire AD, Chaúque HS, et al. (2011) Prozone in malaria rapid diagnostics tests: How many cases are missed? Malar J 10: 166.

20. Luchavez J, Baker J, Alcantara S, Belizario Jr V, Cheng $Q$, et al. (2011) Laboratory demonstration of a prozone-like effect in HRP2-detecting malaria rapid diagnostic tests: Implications for clinical management. Malar J 10: 286.

21. Koita OA, Doumbo OK, Ouattara A, Tall LK, Konare A, et al (2012) False-negative rapid diagnostic tests for malaria and deletion of the histidine-rich repeat region of the hrp2 gene. Am J Trop Med Hyg 86: 194-198.

22. Maltha J, Gamboa D, Bendezu J, Sanchez L, Cnops L, et al. (2012) Rapid diagnostic tests for malaria diagnosis in the Peruvian Amazon: Impact of pfhrp2 gene deletions and cross-reactions. PLoS One 7: e43094.

23. Gatti S, Gramegna M, Bisoffi Z, Raglio A, Gulletta M, et al. (2007) A comparison of three diagnostic techniques for malaria: A rapid diagnostic test (NOW Malaria), PCR and microscopy. Ann Trop Med Parasitol 101: 195-204.

24. Neumann CG, Bwibo NO, Siekmann JH, McLean ED, Browdy B, et al. (2008) Comparison of blood smear microscopy to a rapid diagnostic test for in vitro testing for $P$. falciparum malaria in Kenyan school children. East Afr Med J 85: 544-549.

25. Batwala V, Magnussen $P$, Nuwaha F (2010) Are rapid diagnostic tests more accurate in diagnosis of plasmodium falciparum malaria compared to microscopy at rural health centres? Malar J 9: 349

26. WHO (2016) Malaria microscopy standard operating procedures. Manila: WHO Regional Office for the Western Pacific.

27. Wayne WD (2009) Biostatistics: A Foundation for Analysis in the Health Sciences, John Wiley \& Sons, Atlanta, Ga, USA.

28. Igbeneghu C, Odaibo AB (2012) Plasmodium Species among the Inhabitants of Iwo Community, Southwestern Nigeria. American-Eurasian Journal of Scientific Research 7: 118-122.

29. Ndewa K, Kihamia CM, Mubi M, Semango G (2016) Performance characteristics of malaria rapid diagnostic test and its utilization in management of febrile patients in Korogwe District (Tanga) Tanzania. Universal Journal of Medical Science $4: 81-87$

30. Ajumobi O, Sabitu K, Nguku P, Kwaga J, Ntadom G, et al. (2015) Performance of an HRP-2 rapid diagnostic test in Nigerian children less than 5 years of age. Am J Trop Med Hyg 92: 828-833.

31. Tagbo O, Okafor HU (2007) Comparison of clinical, microscopic and rapid diagnostic test methods in the diagnosis of Plasmodium falciparum malaria in Enugu, Nigeria. Niger Postgrad Med J 14: 285-289.

32. Sani UM, Jiya NM, Ahmed H (2013) Evaluation of malaria rapid diagnostic test among febrile children in Sokoto, Nigeria. Int J Med Sci 3: 434-440.

33. Chika O, Charles J, Emmanuel OS, O Shonekan, V Nwadike, et al. (2016) Histidine rich protein 2 performance in determining the prevalence of Malaria among patients presenting clinical symptoms of malaria. Scientific Journal of Pure and Applied Sciences 5: 339-350.

34. Samane AK, Nahid HZ, Saaed S, et al. (2010) Comparison of microscopy and RDTs technique for laboratory detection of malaria. Afr J Biotechnol 9: 1514-1516.

35. Sheyin Z, Bigwan IE (2013) Comparison of CARE START HRP-2 rapid malaria test with light microscopy for guiding patient's treatment of fever in Nigerian endemic areas. $J$ Med Med Sci 4: 353-356.

36. Nwuba RI, Anumudu Cl, Omosun YO (2004) Evaluation of a rapid test for Plasmodium falciparum. Afr J Med Sci 32: 115-118.

37. Ben-Edet AE, Lesi FEA, Mafe AG, Grange AO (2004) Diagnosis of Plasmodium falciparum malaria in children using the immunochromatographic diagnostic technique. Nig J Paediatr 31: 71-78.

38. Wongsrichanalai C, Barcus MJ, Muth S, Sutamihardja A, Wernsdorfer WH (2007) A review of malaria diagnostic tools: Microscopy and rapid diagnostic test (RDT). Am J Trop Med Hyg 77: 119-127.

39. Wanja EW, Kuya N, Moranga C, Hickman M, Johnson JD, et al. (2016) Field evaluation of diagnostic performance of 
malaria rapid diagnostic tests in western Kenya. Malar $\mathrm{J}$ 15: 456.

40. WHO (2009) Malaria Microscopy Quality Assurance Manual: Version 1. World Health Organization, Geneva.

41. Laferi $H$, Kandel K, Pichler $H$ (1997) False positive dipstick test for malaria. N Engl J Med 337: 1635-1636.

42. Grobusch MP, Alpermann U, Schwenke S, Jelinek T, Warhurst DC (1999) False positive rapid test for malaria in patients with rheumatoid factor. Lancet 353: 297-299.

43. Moody AH, Chiodini PL (2002) Non-microscopic method for malaria diagnosis using OptiMAL IT, a second-generation dipstick for malaria pLDH antigen detection. $\mathrm{Br} \mathrm{J}$ Biomed Sci 59: 228-231.

44. Johanna HK, Christian MT, Inge AJV, Halidou T, Maminata T-C, et al. (2012) Antigen persistence of rapid diagnostic tests in pregnant women in Nanoro, Burkina Faso, and the implications for the diagnosis of malaria in pregnancy. Trop Med Int Health 17: 550-557.

45. Houze S, Boly MD, Le BJ, Deloron P, Faucher J-F (2009) PfHRP2 and PfLDH antigen detection for monitoring the efficacy of artemisinin-based combination therapy (ACT) in the treatment of uncomplicated falciparum malaria. Malar J 8: 211-215.

46. Wellems TE, Walker-Jonah A, Panton LJ (1991) Genetic mapping of the chloroquine- resistance locus on Plasmodium falciparum chromosome 7. Proc Natl Acad Sci USA 88: 3382-3386.

47. Batwala V, Magnussen P, Hansen KS, Nuwaha F (2011) Cost effectiveness of malaria microscopy and rapid diagnostic test versus presumptive diagnosis: Implications for malaria control in Uganda. Malar J 10: 372.

48. Pattanasin S, Proux S, Chompasuk D, Luwiradaj K, Jacquier $P$, et al. (2003) Evaluation of a new Plasmodium lactate dehydrogenase assay (OptiMAL-IT) for the detection of malaria. Trans R Soc Trop Med Hyg 97: 672-674.
49. FMOH (2015) National Guidelines for Diagnosis and Treatment of Malaria: Federal Ministry of Health. Abuja, Nigeria.

50. Warhurst DC, Williams JE (1996) Laboratory diagnosis of malaria. J Clin Pathol 49: 533-538.

51. WHO (2000) Malaria diagnosis, new perspectives. Report of a joint WHO/USAID informal consultation, October 2527. World Health Organization, Geneva.

52. Bell D, Go R, Miguel C, Walker J, Cacal L, et al. (2001) Diagnosis of malaria in a remote area of the Philippines: Comparison of techniques and their acceptance by health workers and the community. Bull World Health Organ 79: 933-941.

53. Aiyenigba B, Ojo A, Aisiri A, Uzim J, Adeusi O, et al. (2017) Immediate assessment of performance of medical laboratory scientists following a 10-day malaria microscopy training programme in Nigeria. Glob Health Res Policy 2: 32.

54. Nankabirwa J, Zurovac D, Niogu JN, Rwakimari JB, Counihan $\mathrm{H}$, et al. (2009) Malaria misdiagnosis in Uganda-implications for policy change. Mal J 8: 66 .

55. Harchut K, Standley C, Dobson A, Klaassen B, Rambaud-Althaus $C$, et al. (2013) Over-diagnosis of malaria by microscopy in the Kilombero Valley, Southern Tanzania: An evaluation of the utility and cost-effectiveness of rapid diagnostic tests. Malaria J 12: 159.

56. Luckett R, Mugizi R, Lopes S, Etossi RC, Allan R (2016) The role of laboratory supervision in improving the quality of malaria diagnosis: A pilot study in Huambo Angola. Am J Trop Med Hyg 94: 659-662.

57. Garmel GM, Mahadevan SV (2012) An introduction to clinical emergency medicine. ( $2^{\text {nd }}$ edn), Cambridge University Press.

58. Lengeler C (2004) Insecticide-treated bed nets and curtains for preventing malaria. Cochrane Database Syst Rev CD000363. 